\title{
Research on the 3D imaging algorithm of spin target based on the Hough transform
}

\author{
Jin Li $i^{*}$ and Yiming Pi
}

\begin{abstract}
As one of the most typical characteristics in space target motion, spin phenomenon has good 3D imaging application potential. Conventional target imaging algorithm fails to make full use of the rotating features of the target to obtain the characteristics of target space, and the speedy spin of targets will cause the dramatic changes in the positions of scattering center within short observation session, which may lead to the failure of imaging algorithm. Aiming at such a special phenomenon of the space target, the time frequency distribution curve of echoes in each scattering center could be mapped onto the parameter space to obtain the position of each scattering center by taking advantage of Hough transformation, thus the 3D features of spin target could be obtained. In this article, the 3D imaging algorithm was studied on the basis of Hough transformation, and its effectiveness was tested with simulation. Meanwhile, the translational motion and shielding effect of space target were discussed, and favorable imaging results were achieved.
\end{abstract}

Keywords: 3D imaging, Spin target, Hough transform, ISAR

\section{Introduction}

Study on the 3D inverse synthetic aperture radar (ISAR) imaging techniques has been attracting more and more attention [1-6]. Compared to 2D ISAR imaging techniques, more detailed information about the target can be provided by 3D ISAR imaging. The current 3D ISAR imaging algorithm mainly consists of two types. The first type takes advantage of various reception channels and receives the echo signals of the target on the basis of phase interference, and it can conduct 2D image for the signals received by each antenna with conventional imaging algorithm [7-9]. As a result, the three-dimensional spatial information of each scattering point can be extracted from the differences of 2D imaging interference phase. In the second type, the target 3D imaging is constructed with the 2D image sequence obtained from different observation angle through a receiving antenna [10]. In both algorithms, a spatial freedom degree is added to obtain three-dimensional resolution.

The traditional ISAR imaging algorithm is based on slow turntable model. High-speed rotating targets, such as airplane propeller, spin precession-guided missile warhead,

\footnotetext{
* Correspondence: flavicidin@vip.163.com

College of Electronic Engineering, University of Electronic Science and Technology of China, Chengdu 611731, China
}

space debris, etc., often fail to meet the requirements of slow turntable model. However, for spin target, its rotating angular velocity can be estimated, which in actually provides a degree of freedom for 3D ISAR imaging [11,12]. As in $[13,14]$, on the condition of the turntable model with high-speed rotating target and its spin angular velocity is known, the methods general radon transform and extended Hough transform were used to threedimensional space information extraction for rotating targets. The basic idea of these algorithms is taking advantage of sine envelope of spin target to estimate the scattering points' three-dimensional location by using curvilinear integral under range-compress domain. The operand of these algorithms is always huge because the energy accumulation along curve is four-dimensional curve detection process. In this article, sine envelope of spin target was used to estimate the 3D position of scattering point in the form of curvilinear integral within distance compressed domain, thus the extraction of 3D spatial information of the spin target could be realized.

\section{Target and echo signal model}

As shown in Figure 1, radar was seated in the origin $O$ of radar coordinate $O-X Y Z$, the projection of radar sight axis on $O X Y$ plane was in the same direction and coincided 


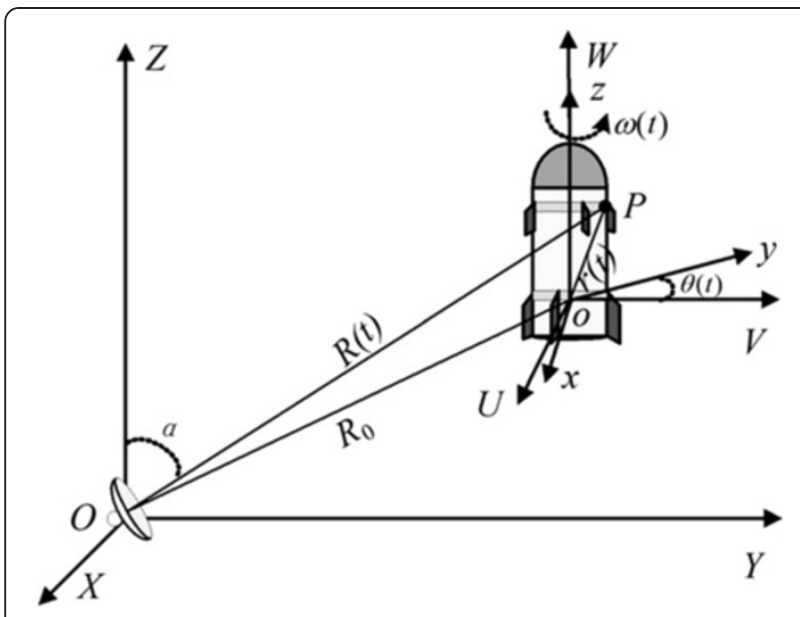

Figure 1 Geometric model for the spatial position relation of spin target.

with $Y$-axis, and the included angle of radar sight axis and $Z$-axis was $\alpha$. Reference frame $o$-UVW paralleled to coordinate system $O-X Y Z$, and both corresponding coordinate axes pointed to the same direction. The origin $o$ was seated in the target rotation axis, axis $W$ was the direction of angular velocity vector of target rotation, axes $U, V$, and $W$ formed right-handed rectangular coordinate system. In $O-X Y Z$ coordinate, the coordinate of point $o$ was $\left(X_{0}, Y_{0}\right.$, $Z_{0}$ ), and obviously $X_{0}=0$. The distance between two coordinate origins $O$ and $o$ was $R_{0}$, and then

$$
\begin{aligned}
& R_{0}=\sqrt{X_{0}^{2}+Y_{0}^{2}+Z_{0}^{2}}=\sqrt{Y_{0}^{2}+Z_{0}^{2}} \\
& \left\{\begin{array}{l}
\sin \alpha=\frac{Y_{0}}{R_{0}}=\frac{Y_{0}}{\sqrt{Y_{0}^{2}+Z_{0}^{2}}} \\
\cos \alpha=\frac{Z_{0}}{R_{0}}=\frac{Z_{0}}{\sqrt{Y_{0}^{2}+Z_{0}^{2}}}
\end{array}\right.
\end{aligned}
$$

The target coordinate system $o-x y z$ and reference coordinate system $o-U V W$ shared the same origin, both the axes $z$ and $W$ pointed to the same direction, and the target coordinate system $o-x y z$ rotated with the targets. Initially, $t_{0}=0$, the target coordinate system $o-x y z$ and reference coordinate system $o-U V W$ coincided, and at moment $t$, the included angle of the two coordinate systems was $\theta(t)$, namely

$$
\theta(t)=\int_{0}^{t} \omega(\tau) d \tau
$$

The coordinate of any scattering point $P$ in coordinate system $o$ - $x y z$ was $\left(x_{P} y_{P}, z_{P}\right)$, while it was $\left(u_{P}, v_{P}, w_{P}\right)$ in coordinate system, obviously

$$
z_{P}=w_{P}
$$

The distance between $P$ and the origin $o$ was $r_{P}$ the coordinate of $P$ in coordinate system $O-X Y Z$ was $\left(X_{B} Y_{P}\right.$ $\left.Z_{P}\right)$, and its distance with origin $O$ was $R_{P}(t)$. Then

$$
\begin{aligned}
R_{P}(t) & =\sqrt{\left(X_{0}+u_{P}\right)^{2}+\left(Y_{0}+v_{P}\right)^{2}+\left(Z_{0}+w_{P}\right)^{2}} \\
& =\sqrt{X_{0}^{2}+Y_{0}^{2}+Z_{0}^{2}+2 X_{0} u_{P}+2 Y_{0} v_{P}+2 Z_{0} w_{P}+u_{P}^{2}+v_{P}^{2}+w_{P}^{2}} \\
& =\sqrt{R_{0}^{2}+2 X_{0} u_{P}+2 Y_{0} v_{P}+2 Z_{0} w_{P}+u_{P}^{2}+v_{P}^{2}+w_{P}^{2}} \\
& =R_{0} \sqrt{1+2 \frac{X_{0}}{R_{0}^{2}} u_{P}+2 \frac{Y_{0}}{R_{0}^{2}} v_{P}+2 \frac{Z_{0}}{R_{0}^{2}} w_{P}+\frac{u_{P}^{2}+v_{P}^{2}+w_{P}^{2}}{R_{0}^{2}}}
\end{aligned}
$$

When the geometric dimensioning of target was far smaller than the distance between the target and radar, namely it met $u_{P}<<R_{0}, v_{P}<<R_{0}$, when $w_{P}<<R_{0}$, Equation (5) could be approximated as the following equation:

$$
\begin{aligned}
R_{P}(t) & \approx R_{0}+\frac{X_{0}}{R_{0}} u_{P}+\frac{Y_{0}}{R_{0}} v_{P}+\frac{Z_{0}}{R_{0}} w_{P} \\
& =R_{0}+\frac{Y_{0}}{R_{0}} v_{P}+\frac{Z_{0}}{R_{0}} w_{P}
\end{aligned}
$$

Project the $3 \mathrm{D}$ figure in Figure 1 onto oUV plane, as shown in Figure 2, then there was the following coordinate transformation relation:

$$
\left\{\begin{array}{l}
u_{P}=x_{P} \cos \theta(t)-y_{P} \sin \theta(t) \\
v_{P}=x_{P} \sin \theta(t)+y_{P} \cos \theta(t)
\end{array}\right.
$$

Substitute Equations (2), (4), and (7) into Equation (6), it could be obtained

$$
\begin{aligned}
R_{P}(t) & \approx R_{0}+\frac{Y_{0}}{R_{0}}\left[x_{P} \sin \theta(t)+y_{P} \cos \theta(t)\right]+\frac{Z_{0}}{R_{0}} z_{P} \\
& =R_{0}+x_{P} \sin \alpha \sin \theta(t)+y_{P} \sin \alpha \cos \theta(t)+z_{P} \cos \alpha
\end{aligned}
$$

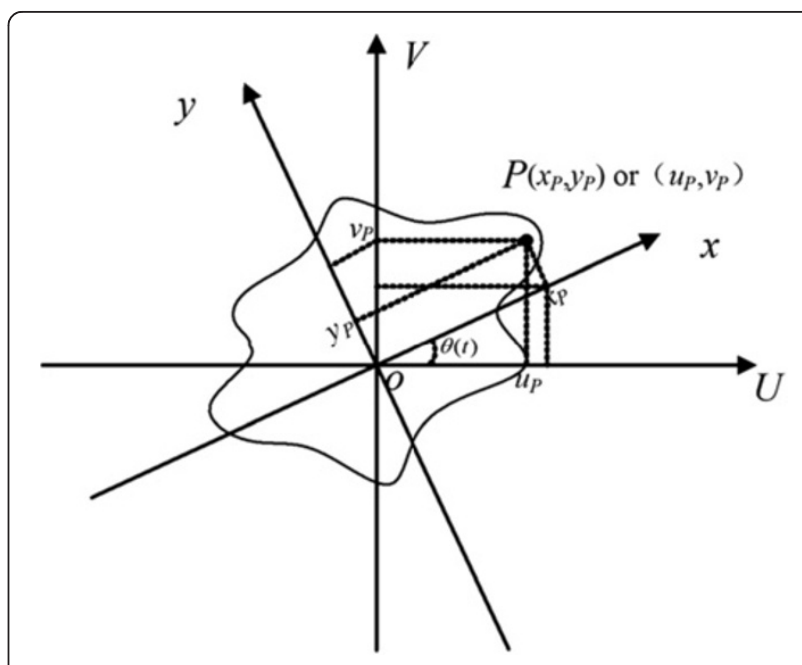

Figure 2 Geometric model for 2D projection of targets. 
Assume the target took uniform rotating motion, namely $\omega(t)=\omega$, was a constant, then

$$
\theta(t)=\omega \cdot t
$$

Substitute it into Equation (8)

$$
\begin{aligned}
R_{P}(t)= & R_{0}+x_{P} \sin \alpha \sin \omega t+y_{P} \sin \alpha \cos \omega t \\
& +z_{P} \cos \alpha
\end{aligned}
$$

To make it simple, assume

$$
\left\{\begin{array}{l}
x_{P} \sin \alpha=x_{P}^{\prime} \\
y_{P} \sin \alpha=y_{P}^{\prime} \\
z_{P} \cos \alpha=z_{P}^{\prime}
\end{array}\right.
$$

then

$$
R_{P}(t)=R_{0}+x_{P}^{\prime} \sin \omega t+y_{P}^{\prime} \cos \omega t+z_{P}^{\prime}
$$

radar linear frequency modulation (LFM) signal

$$
s(\tilde{t})=\operatorname{rect}\left(\frac{\tilde{t}}{T}\right) \exp \left(j 2 \pi f_{0} \tilde{t}\right) \exp \left(j \pi \gamma \tilde{t}^{2}\right)
$$

In which, $T$ is the pulse width, $f_{0}$ is carrier frequency, $\gamma$ is time-frequency rate, $\operatorname{rect}\left(\frac{\tilde{t}}{T}\right)$ is rectangular window function, defined as

$$
\operatorname{rect}\left(\frac{\tilde{t}}{T}\right)=\left\{\begin{array}{l}
1,|\tilde{t}| \leq T / 2 \\
0,|\tilde{t}|>T / 2
\end{array}\right.
$$

The instantaneous frequency of LFM signal was

$$
\begin{aligned}
f & =f_{0}+\gamma \tilde{t},|\tilde{t}| \leq T / 2 \\
& =\operatorname{rect}\left(\frac{\tilde{t}}{T}\right) \cdot\left(f_{0}+\gamma \tilde{t}\right) \\
& =\operatorname{rect}\left(\frac{f-f_{0}}{B}\right) \cdot\left(f_{0}+\gamma \tilde{t}\right)
\end{aligned}
$$

In which, $B=\gamma T$ was the signal bandwidth.

Assume the reflection coefficient of scattering point $P$ within observation time was constant $\sigma_{P}$ then the radar echo of this point could be represented as

$$
s_{P}(f, t)=\sigma_{P} W(f, t) \exp \left[-\frac{j 4 \pi f}{c} R_{P}(t)\right]
$$

In equation $(f, t)=\operatorname{rect}\left(\frac{f-f_{0}}{B}\right) \cdot \operatorname{rect}\left(\frac{t-T_{\mathrm{obs}} / 2}{T_{\mathrm{obs}}}\right), t$ is the observation time, $T_{\text {obs }}$ is the total time-bandwidth of the observation, and $c$ is the speed of light. Substitute
Equation (12) into Equation (16), and compensate for the constant phase term brought by $R_{0}$, it could be obtained

$s_{P}{ }^{\prime}(f, t)=\sigma_{P} W(f, t) \exp \left[-\frac{j 4 \pi f}{c}\left(x_{P}^{\prime} \sin \omega t+y_{P}^{\prime} \cos \omega t+z_{P}^{\prime}\right)\right]$

In radar imaging, the scatterer model was often adopted to describe targets, and the target echo could be seen as the sum of each scattering point echo. For a spin target formed from $K$ scattering points, its corresponding coordinate and reflection coefficient were $\left(x_{k}, y_{k}, z_{k}\right)$ and $\sigma_{k}(k=1,2, \ldots, K)$, then the target echo could be represented as

$$
\begin{aligned}
s(f, t) & =\sum_{k=1}^{K} \sigma_{k} W(f, t) \exp \\
& {\left[-\frac{j 4 \pi f}{c}\left(x_{k} \sin \alpha \sin \omega t+y_{k} \sin \alpha \cos \omega t+z_{k} \cos \alpha\right)\right] } \\
& =\sum_{k=1}^{K} \sigma_{k} W(f, t) \exp \left[-\frac{j 4 \pi f}{c}\left(x_{k}{ }^{\prime} \sin \omega t+y_{k}{ }^{\prime} \cos \omega t+z_{k}{ }^{\prime}\right)\right]
\end{aligned}
$$

Conduct inverse Fourier transform for $f$ in Equation (18), the data after range compression could be obtained, namely the range residence time domain data, as shown in the following equation:

$$
\begin{aligned}
S(r, t) & =\operatorname{IFT}\{s(f, t)\} \\
& =\sum_{k=1}^{K} \sigma_{k} B \cdot \operatorname{rect}\left(\frac{\mathrm{t}-\mathrm{T}_{\mathrm{obs}} / 2}{\mathrm{~T}_{\mathrm{obs}}}\right) \\
& \cdot \operatorname{sinc}\left[\frac{2 \pi B}{\mathrm{c}}\left(\mathrm{r}-\mathrm{x}_{\mathrm{k}}^{\prime} \sin \omega t-\mathrm{y}_{\mathrm{k}}^{\prime} \cos \omega t-\mathrm{z}_{\mathrm{k}}^{\prime}\right)\right] \\
& \cdot \exp \left[\mathrm{j} \frac{4 \pi \mathrm{f}_{0}}{\mathrm{c}}\left(\mathrm{r}-\mathrm{x}_{\mathrm{k}}^{\prime} \sin \omega t-\mathrm{y}_{\mathrm{k}}^{\prime} \cos \omega t-\mathrm{z}_{\mathrm{k}}^{\prime}\right)\right]
\end{aligned}
$$

\subsection{D imaging algorithm}

It could be seen from Equation (19) that, owning to the target spin, the position of range data peak of each scattering point corresponding to the range residence time domain would change according to the range of this scattering point within observation time. Such periodical variation of the peak could be approximated with a sine curve. That is to say, the position of each scattering point in range direction could be approximated as a sine curve, satisfying the following equation:

$$
r=x_{k}{ }^{\prime} \sin \omega t+y_{k}{ }^{\prime} \cos \omega t+z_{k}{ }^{\prime}
$$

On the premise of given sine curve cycle (namely $\omega$ was given), its position and shape were determined by 
three spatial coordinate parameters $x_{k}^{\prime}, y_{k}^{\prime}$, and $z_{k}^{\prime}$. Therefore, the $3 \mathrm{D}$ position information of the scattering point could be obtained by extracting the information about sine curve.

However, it could be seen from the above analysis that, owning to the influence of the included angle $\alpha$ between the radar sight and target axis, the actually extracted position information of scattering point was not authentic position information, but the compression of real position, namely, the position coordinate extracted along the axis direction (namely along axis $z$ ) was about $\cos \alpha$ times of the real position coordinate, while the position coordinate which was vertical to spin axis (namely along axes $x$ and $y$ ) was about $\sin \alpha$ times of the real position coordinate. Therefore, the smaller $\alpha$ was, the closer the coordinate along axis $z$ would be. The better the resolution along axis $z$ was, the worse the resolution along axes $x$ and $y$ would be, and vice versa.

For a target formed from one or several scattering points, the target echo was the sum of each scattering point. Therefore, sine curves might tangle with each other. In addition, with imaging treatment period, each scattering point may not always be shone by the radar beam owning to the shield, namely the scattering point may not have echo. Therefore, there might be discontinuities in sine curve. As a result, it was a little difficult to directly detect the sine curve within range residence time domain, also the image domain. Each sine curve in the image domain corresponded to a peak in the parameter domain, and the parameter corresponding to the peak was the $3 \mathrm{D}$ position coordinate of the scattering point. Consequently, with the help of Hough transformation, the detection for the global curve in the image domain could be converted to detection of peaks in easily realized parameter domain.

The Hough transformation of the image was the cumulates along the transformation curve, defined as follows:

$$
d(\boldsymbol{\Phi})=\sum_{m} D(m, n)=\sum_{m} D(m, f(m ; \boldsymbol{\Phi}))
$$

In the above equation, $d(\Phi)$ was the Hough transformation result of image $D(m, n), \Phi$ was the multidimensional vectors formed by related curve parameters, $n=f(m ; \Phi)$ was the curve to be detected. Here, $m$ was the corresponding residence time $t$, the curve parameter vector $\Phi$ consisted of three positional parameters, namely $x_{k}^{\prime}, y_{k}^{\prime}$, and $z_{k}^{\prime}, f(m ; \Phi)$ corresponded to the curve $r=x_{k}^{\prime} \sin \omega t+y_{k}^{\prime} \cos \omega t+z_{k}^{\prime}$ in the image for range residence time domain.
By equation (19), the image for range residence time domain could be obtained:

$$
G(r, t)=|S(r, t)|
$$

Then its Hough transformation was shown in the following equation

$$
\begin{aligned}
g\left(x_{k}^{\prime}, y_{k}^{\prime}, z_{k}^{\prime}\right) & =\sum_{t} G(r, t) \\
& =\sum_{t} G\left(x_{k}{ }^{\prime} \sin \omega t+y_{k}{ }^{\prime} \cos \omega t+z_{k}{ }^{\prime}, t\right)
\end{aligned}
$$

After Hough transformation, the corresponding curve of each scattering point in range residence time domain would produce a peak in the parameter domain. The estimation for the spatial position of scattering point could easily be realized through detecting the peaks in parameter domain.

Owning to the limited signal bandwidth, there might be certain width in the main lobe of the echo of scattering point after range compression and many side lobes, which would add to the difficulty in actual detection and bring unfavorable influence for imaging, such as the loss of real scattering point, production of fake scattering point, etc. Therefore, the information of each scattering point could be obtained by combining the target parameter estimation method and CLEAN technology. That is to say, after the 3D position coordinate $\left(x_{k}^{\prime}, y_{k}^{\prime}, z_{k}^{\prime}\right)$ was obtained, the point spread function $X(r, t)$ of the scattering point with unit-strength scattering coefficient at this position was constructed according to Equation (19) as follows:

$$
\begin{aligned}
X(r, t) & =B \cdot \operatorname{rect}\left(\frac{\mathrm{t}-\mathrm{T}_{\mathrm{obs}} / 2}{\mathrm{~T}_{\mathrm{obs}}}\right) \\
& \cdot \operatorname{sinc}\left[\frac{2 \pi B}{\mathrm{c}}\left(\mathrm{r}-\mathrm{x}_{\mathrm{k}}{ }^{\prime} \sin \omega t-\mathrm{y}_{\mathrm{k}}{ }^{\prime} \cos \omega t-\mathrm{z}_{\mathrm{k}}{ }^{\prime}\right)\right] \\
& \cdot \exp \left[\mathrm{j} \frac{4 \pi \mathrm{f}_{0}}{\mathrm{c}}\left(\mathrm{r}-\mathrm{x}_{\mathrm{k}}{ }^{\prime} \sin \omega t-\mathrm{y}_{\mathrm{k}}{ }^{\prime} \cos \omega t-\mathrm{z}_{\mathrm{k}}{ }^{\prime}\right)\right]
\end{aligned}
$$

According to certain standard and taking advantage of $S(r, t)$ and $X(r, t)$, the scattering coefficient of scattering point in this position could be estimated.

For example, the estimation of scattering coefficient could select the following minimum norm criterion:

$$
\begin{aligned}
\hat{\sigma} & =\arg \min _{\sigma} I(\sigma) \\
& =\arg \min _{\sigma}\|S(r, t)-\sigma X(r, t)\| \\
& =\arg \min _{\sigma} \sum_{r, t}|S(r, t)-\sigma X(r, t)|^{2}
\end{aligned}
$$




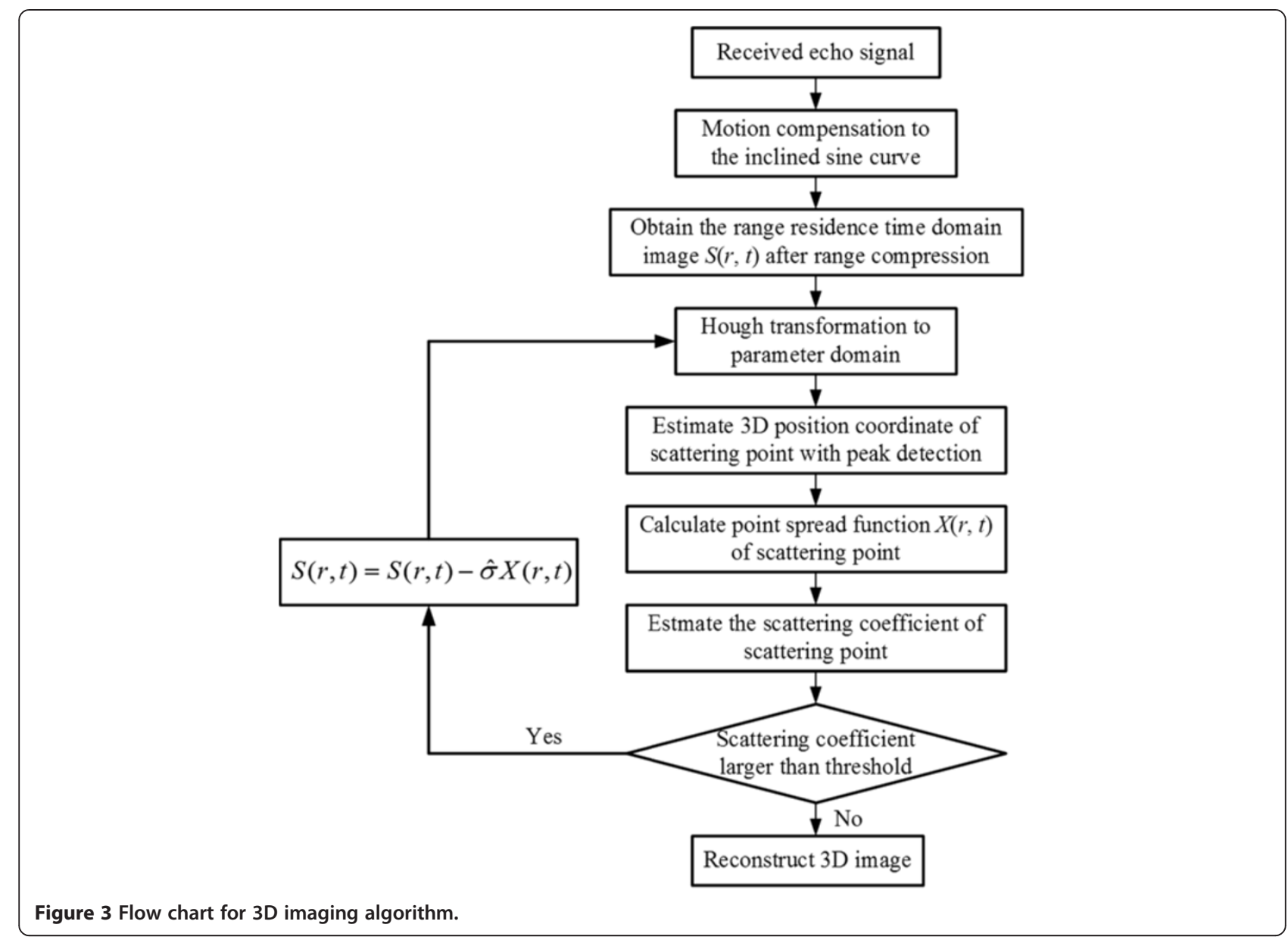

In order to solve Equation (25), adopt derivative about $\sigma$ from $I(\sigma)$, and suppose it as 0 , namely

$$
\frac{\partial I(\sigma)}{\partial \sigma}=-2 \sum_{r, t}[S(r, t)-\sigma X(r, t)] X^{*}(r, t)=0
$$

Table 1 Coordinate and scattering coefficient of each scattering point

\begin{tabular}{lllll}
\hline \multirow{2}{*}{ Number } & \multicolumn{2}{c}{ Coordinate of 3D position } & \multirow{2}{*}{$\begin{array}{l}\text { Scattering } \\
\text { coefficient } \boldsymbol{\sigma}\end{array}$} \\
\cline { 2 - 4 } & $\boldsymbol{x}(\mathrm{m})$ & $\boldsymbol{y}(\mathrm{m})$ & $\boldsymbol{z}(\mathrm{m})$ & \\
\hline $0.3 \sqrt{2}$ & 0 & $-0.6 \sqrt{2}$ & 1.3 \\
\hline 3 & 0 & $0.3 \sqrt{2}$ & $-0.6 \sqrt{2}$ & 1.0 \\
\hline 4 & $-0.3 \sqrt{2}$ & 0 & $-0.6 \sqrt{2}$ & 1.0 \\
\hline 5 & 0 & $-0.3 \sqrt{2}$ & $-0.6 \sqrt{2}$ & 1.0 \\
\hline 6 & $0.2 \sqrt{2}$ & $0.2 \sqrt{2}$ & $0.3 \sqrt{2}$ & 1.3 \\
\hline 7 & $-0.2 \sqrt{2}$ & $0.2 \sqrt{2}$ & $0.3 \sqrt{2}$ & 1.0 \\
\hline 8 & $-0.2 \sqrt{2}$ & $-0.2 \sqrt{2}$ & $0.3 \sqrt{2}$ & 1.0 \\
\hline 9 & $0.2 \sqrt{2}$ & $-0.2 \sqrt{2}$ & $0.3 \sqrt{2}$ & 1.0 \\
\hline
\end{tabular}

In which, $X^{*}(r, t)$ was the conjugation of $X(r, t)$. Then the estimation value $\hat{\sigma}$ of scattering coefficient could be obtained

$$
\hat{\sigma}=\frac{\sum_{r, t} S(r, t) X^{*}(r, t)}{\sum_{r, t}|X(r, t)|^{2}}
$$

After the information about 3D position and scattering coefficient of this scattering point was obtained, the information about this scattering point in the echo data was eliminated, namely suppose $S(r, t)=S(r, t)-\hat{\sigma} X(r, t)$, and the above procedures of parameter estimation were repeated with new data $S(r, t)$, till $\hat{\sigma}$ was smaller than the pre-set threshold. At this moment, the information of all scattering points was extracted from echo data, and 3D image of the target could be reconstructed with the information.

In practice, sinc function was characterized with certain main lobe width, and together with the interference of echo side lobe of other scattering points and the influence of parameter discrete step in parameter domain, there might be differences between the parameters extracted in the 
parameter domain after Hough transformation and actual coordinate. At this moment, the coordinate value with errors were adopted for constructing point spread function, then there might be relatively large errors in the scattering coefficient obtained, which would influence the performance of CLEAN algorithm and lead to the decrease in the performance of 3D imaging algorithm. In order to improve the accuracy of the estimation for the position of scattering point and scattering coefficient, the following thought could be adopted: the estimated value of the information of scattering point was obtained through Hough transformation, the estimated value was adopted as the initial value, and then parameters satisfying Equation (28) were searched with small step around the scattering point, which would be regarded as the final estimated value of the scatterer parameter.

$$
\begin{aligned}
& \left(\hat{x}_{k}^{\prime}, \hat{y}_{k}{ }^{\prime}, \hat{z}_{k}{ }^{\prime}, \hat{\sigma}\right)=\arg \min _{x_{k}^{\prime}, y_{k}^{\prime} z_{k} \sigma}\|S(r, t)-\sigma X(r, t)\| \\
& =\arg \min _{x_{k^{\prime}, y_{k}^{\prime}, z_{k} \cdot \sigma}}\left\|S(r, t)-\sigma X\left(x_{k}^{\prime}, y_{k}^{\prime}, z_{k}^{\prime}, t\right)\right\|
\end{aligned}
$$

The specific flow chart for 3D imaging algorithm was shown in Figure 3.

\section{Simulation experiment}

The signal bandwidth was $4 \mathrm{GHz}$, while the time bandwidth was $4 \mu \mathrm{s}$. The pulse repetition frequency was 0.6 $\mathrm{kHz}$. Assume that the included angle of radar sight and target spin axis was $\pi / 4$, the distance between the target center and radar was about $14.14 \mathrm{~km}$, the angular velocity of the target spin was $5 \mathrm{~Hz}$. In this equation, the translation component of the target should be compensated well to make sure that the included angle of the radar sight and spin axis should remain unchanged when the target spun in high speed. Radar far-field target formed by nine scattering point shall be established as shown in Table 1, in addition, this target also had velocity component $v_{e}=1 / s$ along $z$ direction.

The image of range residence time domain was shown in Figure 4, and obviously owing to the velocity component in $z$ direction, the position of scattering points changed continuously. As a result, the center of sine curve also changed continuously, namely 'inclined' sine curve was formed.

In order to make up for the translation component, the 'inclined' sine curve could be corrected. And the curve expression could be adjusted during Hough transformation, namely to substitute $z_{k}^{\prime}$ with $z_{k}^{\prime}+v_{e} t$ in Equation (23), the corresponding Hough transformation could be changed to the following form:

$$
\begin{aligned}
g\left(x_{k}^{\prime}, y_{k}^{\prime}, z_{k}^{\prime}, v_{e}\right) & =\sum_{t} G(r, t) \\
& =\sum_{t} G\left(x_{k}^{\prime} \sin \omega t+y_{k}^{\prime} \cos \omega t+z_{k}^{\prime}+v_{e} t, t\right)
\end{aligned}
$$

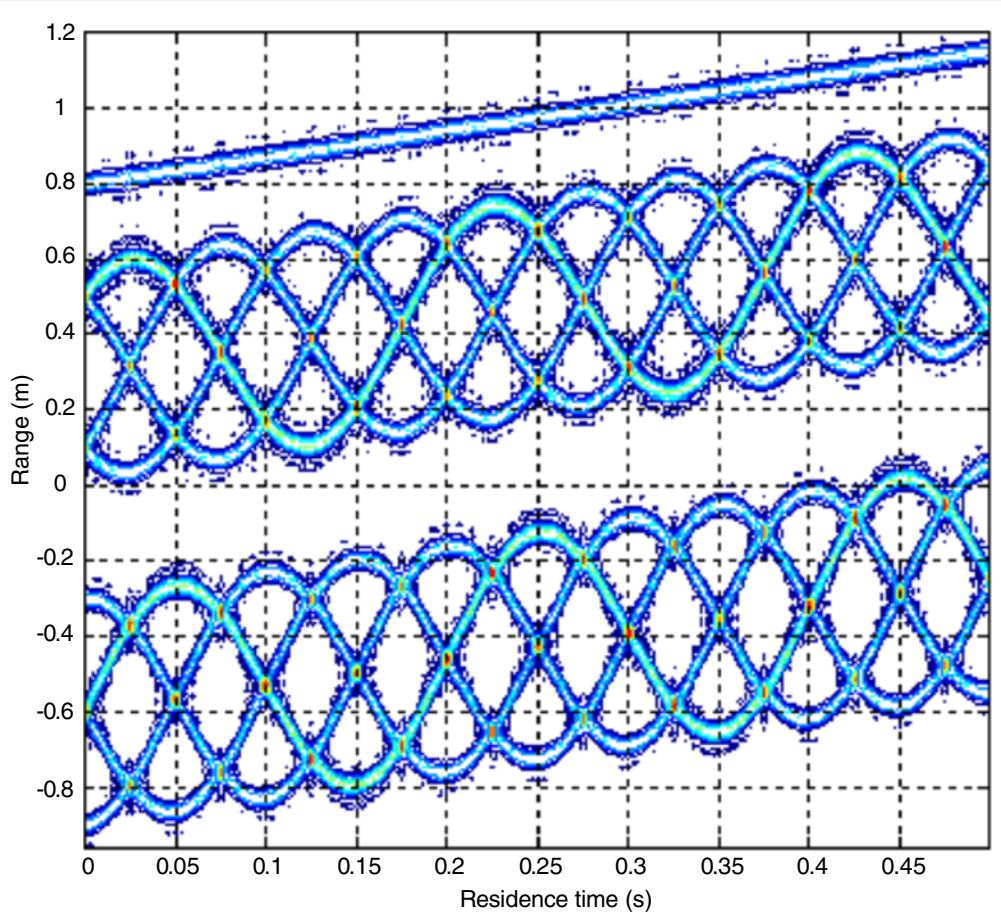

Figure 4 The image of range residence time domain under translation condition. 


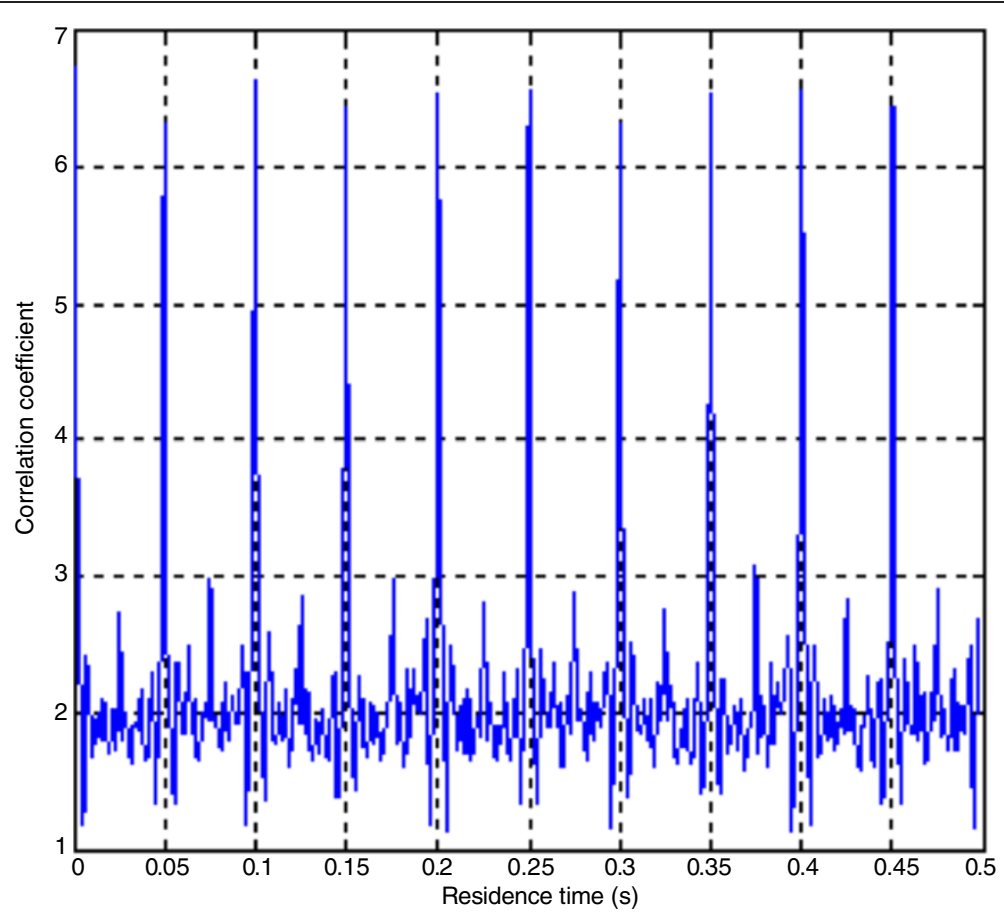

Figure 5 The maximum correlation coefficient of echo at $t=0$ and other moments.

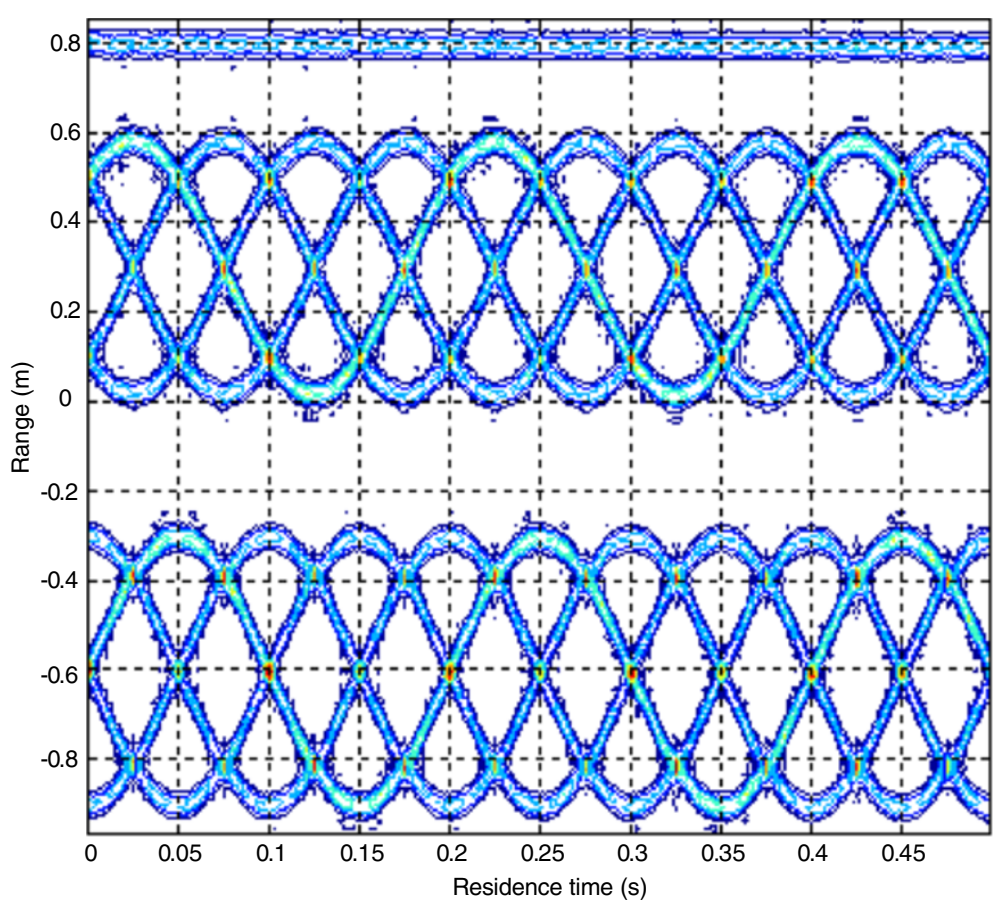

Figure 6 The image of range residence time domain after compensating the translation component. 


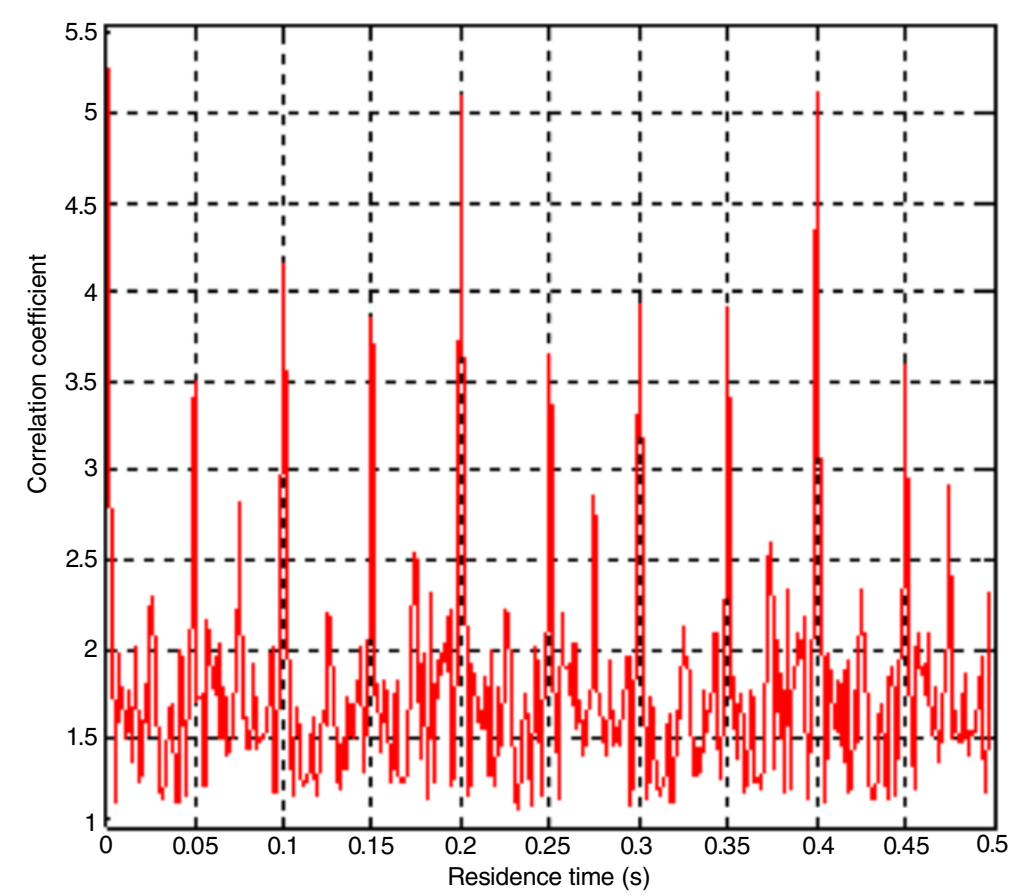

Figure 7 The maximum the maximum correlation coefficient of echo at $t=0$ and other moments.

One or several scattering points could be used to obtain the estimation value $\hat{v}_{e}$ of translational velocity, which would compensate for the translation component caused by this speed. However, the original 3D parameter domain $\left(x_{k}^{\prime}, y_{k}^{\prime}, z_{k}^{\prime}\right)$ would be added to $4 \mathrm{D}$ parameter domain $\left(x_{k}^{\prime}, y_{k}^{\prime}, z_{k}^{\prime}, v_{e}\right)$. When estimating the parameter, 4D search was needed. Therefore, this method would greatly increase the calculated amount.

In order to decrease the calculated amount, slide correlation-based method could be adopted. According

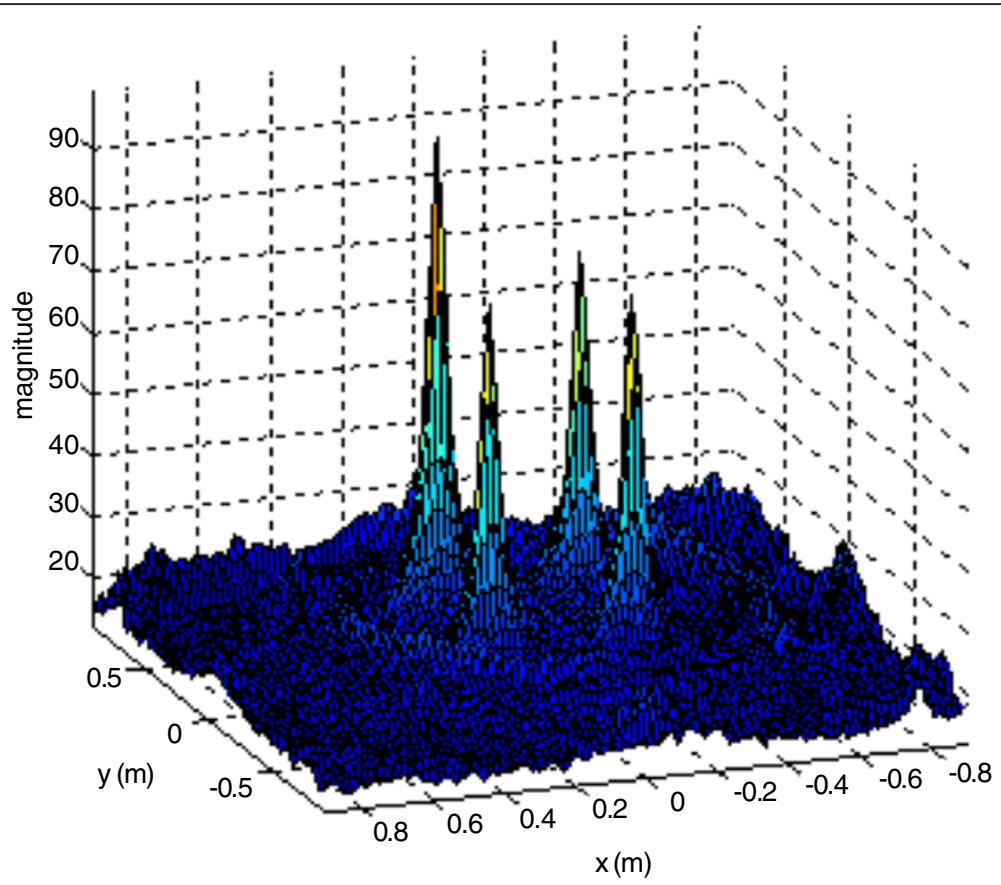

Figure 8 The results of parameter domain after Hough at point $(z=-0.6 \mathrm{~m})$ in plane $z$. 


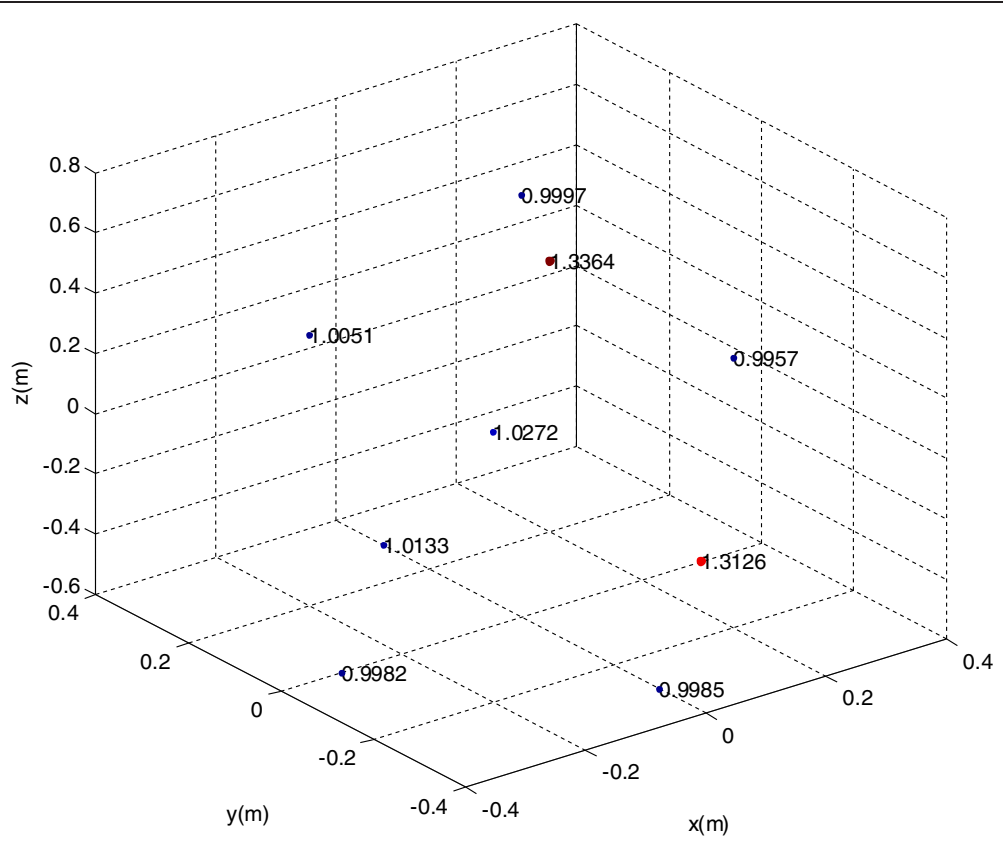

Figure 9 Result of 3D imaging.

to the position of maximum correlation coefficient, the translation component could be compensated. In Figure 5, the maximum correlation coefficient of the first echo (namely echo at $t=0$ ) and other echoes. Owning to the good symmetry among each scattering point, the peak would occur at the quarter of each rotation cycle. The range residence time domain after compensation was shown in Figure 6, and obviously the translation component was compensated well. At this moment, subsequent algorithm such as Hough transformation, etc., could be conducted, and 3D imaging could be realized.

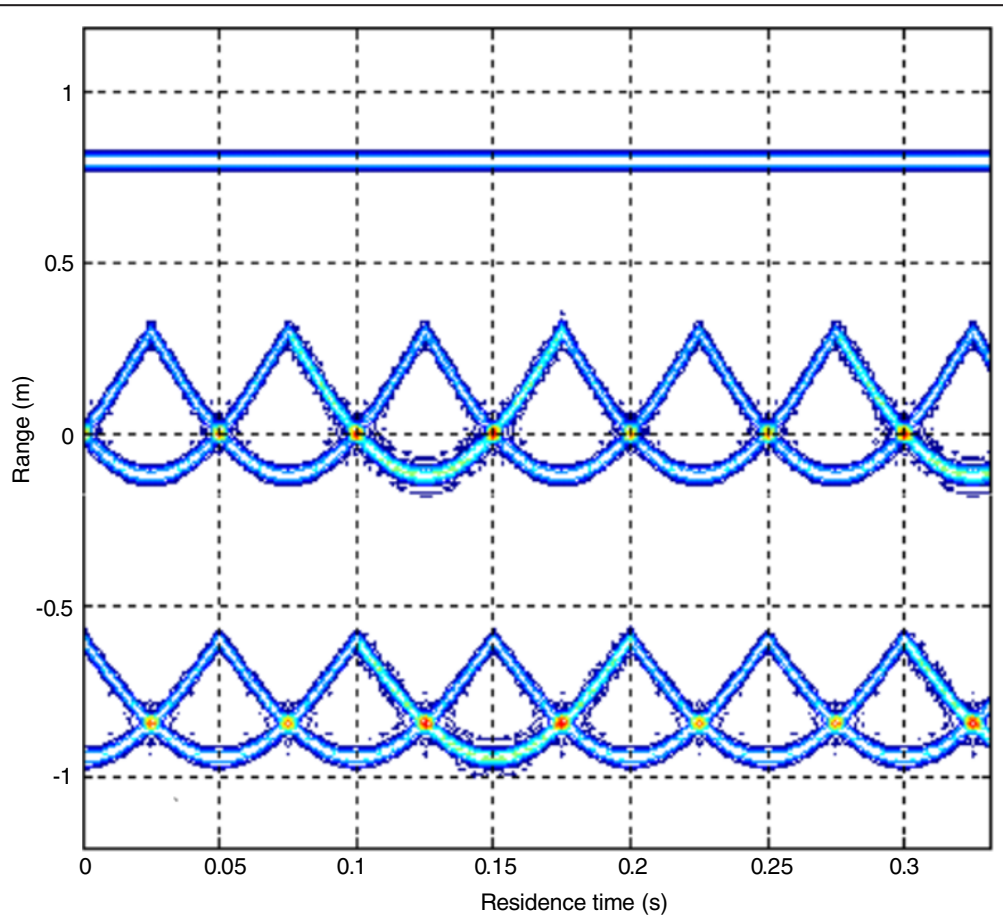

Figure 10 The range residence time domain image of multi-scattering points after taking shield into consideration. 


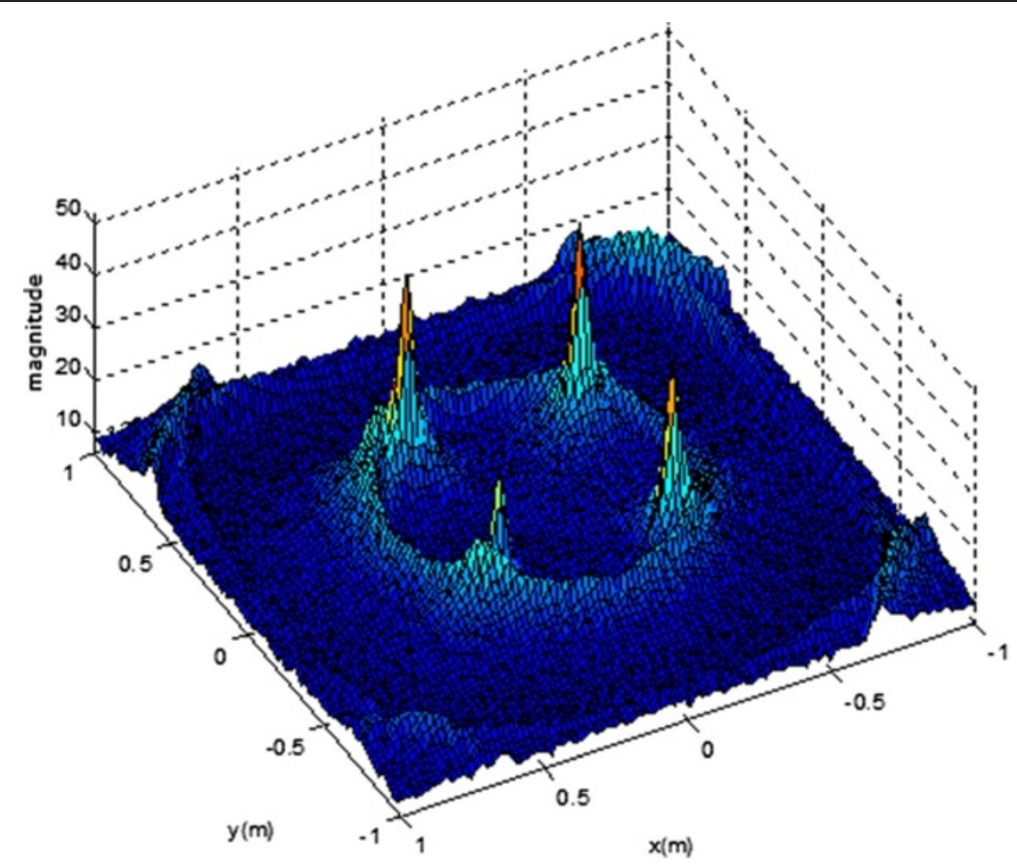

Figure 11 The results of parameter domain after Hough at point $(z=0.3 \sqrt{2} \mathrm{~m})$ of plane $z$ after taking shield into consideration.

When there was a lack of good symmetry in each target scattering point, if only seven scattering points were selected, such as the (1)(2)(3(5)(6)(7) in Table 1, the maximum correlation coefficient of echo at $t=0$ and other moments were shown in Figure 7. Obviously, the maximum peak would occur only when the rotation cycle was in integral multiple. For the unknown rotation cycle, this method could also be used to estimate the spin cycle of the target.

As shown in Figure 6, the image of range residence time domain was transferred to parameter domain after Hough transformation, the result was cut out at $z=$ -0.6 , as shown in Figure 8. There were four scattering points in this plane, therefore, it would form four

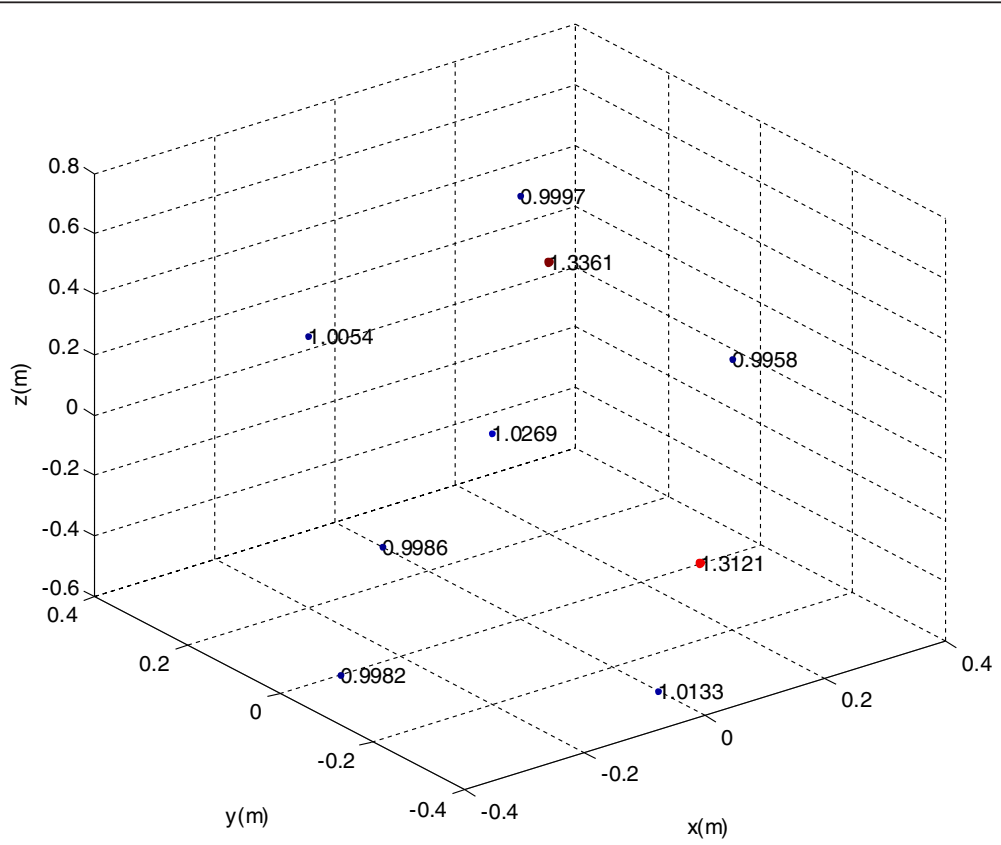

Figure 12 Results of 3D imaging after taking shield into consideration. 
distinct peaks in the parameter domain, and the corresponding parameter information was the spatial position information of the scattering point. Besides, owning to the influences of such factors as the side lobes of each scattering point, there were small fluctuations in the parameter domain.

The final 3D imaging result was shown in Figure 9. Since the radar sight angle $\alpha=\pi / 4$, the coordinate value in the direction of axes $x, y$, and $z$ was about $\sqrt{2} / 2$ times of the real value. In the figure, the marked figures were the estimated value of the scattering coefficient of the scattering point at corresponding position obtained from this algorithm. It could be seen from the figure that $3 \mathrm{D}$ imaging could be realized from this algorithm.

Since it was unavoidable that the scattering center of the spin target was seated in the back of radar sight, it was impossible for each scattering center to be shone by the radar beam. Therefore, the shielding condition in the scattering center should be considered. The range residence time domain image after taking shield into consideration and transformational results of parameter domain were shown in Figures 10 and 11, respectively, in which the parameter of each scattering point was shown in Table 1. Within image observation period, scattering point (9) on the spin axis could always be seen, while the rest eight ones could be seen within half cycle close to radar. It could be seen from Figure 10 that owning to the influence of shield, the sine curve in the image domain was discontinuous, and at this moment, it was difficult to directly detect the curve. There was obvious peak in the parameter domain shown in Figure 11, which was easier to detect the parameter.

The results of 3D imaging after taking shield into consideration were shown in Figure 12, and obviously, it could also obtained good imaging results.

\section{Conclusion}

In this article, 3D imaging algorithm of spin target based on Hough transformation was proposed. By taking advantage of the sine envelope of spin target, the $3 \mathrm{D}$ position of scattering point was estimated in the form of curve integral within range compression domain, thus the extraction of 3D spatial information of the spin target could be realized, and this algorithm was tested to be valid through simulation experiment. In addition, the translational motion and shielding condition existed in the real target were also taken into consideration in the simulation experiment, and corresponding measures were adopted for translational motion compensation. Under the circumstance of incomplete target sine curve, the target parameter was extracted to realize the $3 \mathrm{D}$ imaging of spin target.

The authors declare that they have no competing interests.

\section{Acknowledgement}

This study was supported by the National Natural Science Foundation of China (61271287) and the Fundamental Research Funds for the Central Universities (ZYGX2011J020).

Received: 5 December 2012 Accepted: 28 February 2013 Published: 28 March 2013

References

1. VC Chen, F Li, SS Ho et al., Analysis of micro-Doppler signatures. IEE Proc. Radar Sonar Navigat. 150(4), 271-276 (2003)

2. Q Zhang, TS Yeo, G Du, SH Zhang, Estimation of three dimensional motion parameters in interferometric ISAR imaging. IEEE Trans. Geosci. Remote Sens. 42(2), 292-300 (2004)

3. J Tsao, BD Steinberg, Reduction of sidelobe and speckle artifacts in microwave imaging: the CLEAN technique. IEEE Trans. Antennas Propagat. 36(4), 543-556 (1988)

4. T Sparr, B Krane, Micro-Doppler analysis of vibrating targets in SAR. Proc. Inst. Electr. Eng.-Radar Sonar Navigat. 150(4), 277-283 (2003)

5. J Li, YM Pi, Micro-Doppler signature feature analysis in terahertz band. J. Infrared Millim. Terahertz Waves 31(3), 319-328 (2010)

6. J Li, H Ling, Application of adaptive chirplet representation for ISAR feature extraction from targets with rotating parts. Proc. Inst. Electr. Eng.-Radar Sonar Navigat. 150(4), 284-291 (2003)

7. GY Wang, XG Xia, VC Chen, Three-dimensional ISAR imaging of maneuvering targets using three receivers. IEEE Trans. Image Process. 10(3), 436-448 (2001)

8. XJ Xu, RM Narayanan, Three-dimensional Interferometric ISAR imaging for target scattering diagnosis and modeling. IEEE Trans. Image Process. 10(7), 1094-1102 (2001)

9. JT Mayhan, ML Burrows, KM Cuomo, JE Piou, High resolution 3D "snapshot" ISAR imaging and feature extraction. IEEE Trans. Aerosp. Electron. Syst. 37(2), 630-642 (2001)

10. JL Walker, Range-Doppler imaging of rotating objects. IEEE Trans. Aerosp. Electron. Syst. AES-16(1), 23-52 (1980)

11. KV Hansen, PA Toft, Fast curve estimation using preconditioned generalized radon transform. IEEE Trans. Image Process. 5(12), 1651-1661 (1996)

12. PA Toft, Using the generalized radon transform for detection of curves in noisy images, IEEE International Conference on Acoustics, Speech and Signal Processing, Atlanta, USA, vol. IV, 1996, pp. 2219-2222

13. Q Wang, MD Xing, High-resolution three-dimensional radar imaging for rapidly spinning targets. IEEE Trans. Geosci. Remote Sens. $46(1), 22-30(2008)$

14. Q Zhang, TS Yeo, Imaging of a moving target with rotating parts based on the Hough transform. IEEE Trans. Geosci. Remote Sens. 46(1), 291-299 (2008)

doi:10.1186/1687-1499-2013-90

Cite this article as: $\mathrm{Li}$ and Pi: Research on the 3D imaging algorithm of spin target based on the Hough transform. EURASIP Journal on Wireless Communications and Networking 2013 2013:90.

\section{Submit your manuscript to a SpringerOpen ${ }^{\circ}$ journal and benefit from:}

- Convenient online submission

- Rigorous peer review

- Immediate publication on acceptance

- Open access: articles freely available online

- High visibility within the field

- Retaining the copyright to your article

Submit your next manuscript at $>$ springeropen.com 